\title{
LOS VIDEOJUEGOS EN ESPAÑA: UNA INDUSTRIA CULTURAL INCIPIENTE
}

\author{
Vanessa Rodríguez Breijo \\ Universidad San Jorge \\ vrodriguez@usj.es \\ José Manuel Pestano Rodríguez \\ Universidad de La Laguna \\ jpestano@ull.es
}

\begin{abstract}
Resumen: La situación actual de la industria del videojuego en España se caracteriza por las tendencias oligopólicas del sector, la integración vertical de las multinacionales dominantes, la insuficiente financiación pública a las desarrolladoras de software nacionales y la carencia de inversión en $I+D$, factores que han tenido como consecuencia que las empresas foráneas (especialmente norteamericanas y japonesas) estén cubriendo más del $94 \%$ de la demanda. En este artículo se describe cómo se configura, desde una perspectiva de mercado, la industria de ocio audiovisual que alcanza la mayor facturación en este país.
\end{abstract}

Palabras claves: Videojuegos, industria cultural, empresas desarrolladoras, contenidos digitales.

Abstract: The current state of the video game industry in Spain is characterized by the oligopolistic trends of the industry, the vertical integration of the leading multinationals, the insufficient public funding of national software development and the lack of investment in $R \& D$, which has allowed foreign companies (especially American and Japanese) to cover more than $94 \%$ of the demand. This article describes the configuration, from a market perspective, of the audiovisual entertainment industry with the highest turnover in this country.

Key Words: Video games, culture industry, development companies, digital content..

\section{INTRODUCCIÓN}

A comienzos del siglo XXI los videojuegos se configuran como un sector relevante dentro de las industrias culturales, suscitando el interés de los usuarios, estableciendo relaciones con otros medios desde una posición dominante y sumando adeptos y detractores ante la mirada de los académicos e investigadores, algunos todavía sorprendidos por la velocidad del fenómeno. El campo de los 
videojuegos constituye ahora un territorio de estudio novedoso y fecundo en el que intervienen numerosas disciplinas empleando teorías, metodologías y puntos de vista diferentes.

Los videojuegos expanden su área de influencia más allá de su ámbito específico y establecen relaciones relevantes con otros medios, como la televisión (Evans, 2008), el cine (Grusin, 2006) y la industria editorial (Pestano et al. 2010); por su parte, la prensa les dedica espacio y tiempo en forma de apartados específicos y piezas de actualidad; los juegos se convierten en un vector novedoso para contenidos aparentemente distantes de su objetivo lúdico como la publicidad (Kuhn et al., 2007; Martí Parreño, 2010), el aprendizaje (BARANOWSKI et al., 2005; Gros y Bernat, 2008; Hayes y Games, 2008), la salud (Baranowski et al., 2008) y el entrenamiento para la resolución de problemas reales (Sánchez et al., 2009; Zyda, 2005). En el ámbito académico, poco a poco, los videojuegos configuran un territorio multidisciplinar en el que también tiene presencia la comunicación (Aarset, 1997).

En términos de industria cultural, se estudian los videojuegos a través de comparaciones internacionales que permiten establecer la situación de determinadas características del sector en diferentes países (Okazaki et al., 2007; Teipen, 2008); también se ha observado la utilización de las redes para la producción y distribución de videojuegos (Johns, 2005), así como las implicaciones de la globalización en las organizaciones productivas (Consalvo, 2006). En otros casos se prefiere profundizar en la dinámica económica de los videojuegos en un país concreto (Storz, 2008), contexto en el que se enmarca este trabajo, sin olvidar que la complejidad de este producto cultural remite insistentemente a las dimensiones reseñadas más arriba. Esta elección se fundamenta en resultados precedentes de la investigación comparada (Izushi y Aoyama, 2006) que indican que la evolución del sector en un país concreto obedece a determinadas condiciones preexistentes del entorno, a su disposición innovadora y a su cultura.

En España el mercado de los videojuegos se ha visto influido por la formación y consolidación de grupos multinacionales que concentran las cuotas más importantes de la demanda. Esta tendencia internacional ha marcado el desarrollo de la industria de la comunicación desde los años 90 y no es exclusiva del sector de los games. Se manifiesta en la escasa cantidad de actores que operan en el mercado y en el crecimiento de estos en detrimento de las empresas con menor músculo financiero, que han sido incapaces de asumir el reto de la internacionalización.

Este proceso ha sido consecuencia, por una parte, de esa necesidad de proyectar los productos más allá de las fronteras, que requería grandes inversiones y el acceso al mercado de capitales. La dependencia de este, entre otros factores, ha ocasionado fusiones entre importantes grupos y un progresivo aumento de la concentración. Por otra parte, esta situación no se habría podido producir sin el relajamiento de las políticas anti monopólicas, producto de la aplicación de medidas liberales a nivel mundial. En este sentido, la defensa de la economía y la cultura nacionales en mercados globales ha pasado casi en su totalidad a 
manos privadas (Aguado et. al, 2008).

En este contexto tienen lugar distintas estrategias de concentración empresarial. La integración vertical es una de ellas y se basa en el control de las diferentes fases del proceso de producción y comercialización de un bien por una misma corporación (Sánchez-Tabernero, 2000). Es ascendente cuando la empresa comienza a controlar una fase posterior a la que le era propia (por ejemplo, la distribución), y descendente cuando establece su control en una fase anterior (como es el caso del suministro). Por otro lado, la estrategia de la integración multimedia hace referencia la presencia de un grupo en distintos canales o medios de comunicación, para ganar una cuota mayor de mercado y aprovechar las sinergias y la diversificación de riesgos. Esa estrategia es similar a la multisectorial, aunque en esta última existe una participación en empresas de distintos ámbitos, no solo de la comunicación. La integración internacional, finalmente, configura un escenario en el que las economías de escala permiten la presencia de un grupo en distintos países, aprovechando el know-how de cada ubicación geográfica (Aguado et. al, 2008).

Estas tres estrategias condicionan la importante presencia de los grupos multinacionales -especialmente norteamericanos y japoneses- en el mercado español de los videojuegos, así como la escasa participación de las empresas españolas en la oferta. En este artículo se pretende demostrar cómo esta concentración empresarial multinacional, unida a las particulares características de un producto que requiere de grandes inversiones y riesgos, y a un escaso apoyo estatal al sector, configuran un panorama en el que la mayor parte de la demanda nacional (un 94\%) está siendo satisfecha por productos internacionales, mientras que las empresas españolas apenas logran seguir a flote. Todo ello en un contexto en el que cada día crecen los aficionados a los videojuegos, y las ganancias obtenidas por venta de juegos y consolas casi no se han resentido por efectos de la crisis económica.

De este modo, se ha dividido la investigación en dos partes, correspondientes respectivamente a la oferta y la demanda en el mercado español de los videojuegos. En la primera parte (epígrafes 2 y 3), se describe el crecimiento de la demanda nacional, caracterizada por ser la cuarta más importante de la Unión Europea y por expandirse a distintos sectores sociales que antes eran ajenos a esta forma de entretenimiento. Igualmente, se verifica que el crecimiento del sector es mayor al experimentado por el cine, el DVD y la música.

En la segunda parte (epígrafes 4, 5, 6 y 7), se estudia la configuración de la oferta de videojuegos en España. A través de los datos disponibles sobre el escaso tamaño y facturación de las desarrolladoras nacionales, por una parte, y sobre el dominio de los grupos multinacionales en los rankings de ventas, por otra, se comprueba que el mercado tiene una configuración oligopólica, con una preponderancia de los productos foráneos. Se verifica también que los grandes grupos dominantes del mercado utilizan las estrategias de integración vertical, 
multimedia, multisectorial e internacional.

Pero la posición dominante de los grupos multinacionales no es la única responsable de este rezago en la industria nacional del videojuego, por ello también se estudian en esta segunda parte las carencias que existen en el otorgamiento de ayudas estatales a desarrolladoras nacionales. Para ello se comparan las subvenciones concedidas por el Ministerio de Cultura a los sectores editorial, cinematográfico, audiovisual y de los videojuegos. También se indaga en la inversión que se está destinando a la investigación y desarrollo en software de entretenimiento en España, aspecto fundamental para poder posicionarse en un mercado exigente en materia de innovación y tecnología. La oferta formativa y laboral de los desarrolladores y la repercusión de la piratería fueron los dos últimos temas que se abordaron, pues no puede obviarse la repercusión que tienen en la competitividad de la oferta nacional.

\section{UNA DEMANDA ASCENDENTE Y PROMETEDORA}

En el año 2010 los videojuegos hicieron, en el ámbito mundial, una caja de 56.000 millones de euros, superando en más de tres mil millones la facturación de 2009 (Adese, 2011a). Entre 2007 y 2008 fueron la industria generadora de contenidos digitales que más aumentó su facturación (un 15\%) en todo el planeta, después de la publicidad online (Gfk Emer Ad-hoc research, 2010).

En el mercado mundial, España es el sexto país con mayor facturación y en el europeo es el cuarto, después de Reino Unido, Francia y Alemania (Adese, 2011a). También ocupa esta posición en lo que respecta a número de jugadores y a parque de dispositivos existente (4,96 millones de equipos) (AETIC, 2011).

Un $24 \%$ de la población española utiliza habitualmente videojuegos (Adese, 2011a). Si solo se cuenta a quienes tienen edades entre siete y 34 años, el porcentaje se eleva a 45,3\% (Adese, 2011b). Desde 2004 hasta 2009 el grupo que más ha aumentado proporcionalmente el uso de videojuegos tanto de consola como de ordenador ha sido el de niños de siete a trece años, estableciéndose como los que más juegan: más del $80 \%$ de la población con estas edades son gamers. Sin embargo, cada vez hay más jugadores adultos. Casi uno de cada cuatro españoles mayores de 65 años utiliza videojuegos por lo menos una vez al mes, según datos de un estudio realizado por Universidad Complutense de Madrid y la Asociación de videojugadores (2008). Asimismo, la mitad de las personas con edades entre 45 a 54 años tiene esa misma frecuencia de juego.

La venta de consolas alcanzó en 2010 una cifra de 489 millones de euros y la de software de entretenimiento 631 millones. Así, los videojuegos se consolidan como la actividad de consumo de ocio audiovisual e interactivo que más dinero genera, alcanzando en total la cifra de 1.120 millones de euros ese año (Adese, 2011a). El volumen de ganancias creadas por los videojuegos ha ido en franco aumento, siendo en 2010 más de dos veces mayor que en el 2000, como puede 
apreciarse en el siguiente cuadro.

Cuadro 1. Evolución del mercado de los videojuegos en España 2000-2010.

\begin{tabular}{|l|c|}
\hline Año & $\begin{array}{c}\text { Facturación del sector de los } \\
\text { videojuegos (millones de } € \text { ) }\end{array}$ \\
\hline 2000 & 504,80 \\
\hline 2001 & 579,98 \\
\hline 2002 & 710,03 \\
\hline 2003 & 800,13 \\
\hline 2004 & 790,27 \\
\hline 2005 & 863,00 \\
\hline 2006 & 967,00 \\
\hline 2007 & $1.454,00$ \\
\hline 2008 & $1.432,00$ \\
\hline 2009 & $1.200,00$ \\
\hline 2010 & $1.120,00$ \\
\hline
\end{tabular}

Fuente: Datos de Adese recogidos en Pérez Martín, Diego Martínez y Parra Valcarce (2009) y actualizado con datos de Adese (2010 y 2011a).

Las ganancias de esta industria crecieron muy rápidamente hasta el año 2007, disminuyendo levemente en 2008, 2009 y 2010 (un 1,5\%, un 16\% y un 6,6\% respectivamente) por efectos de la desaceleración económica. No obstante, este declive ha sido mucho mayor $-\mathrm{y}$ anterior a la crisis- en otros sectores audiovisuales y de la cultura, siendo especialmente notorio en el mercado del DVD, la música grabada y el cine (Checa Godoy, 2009). En el siguiente cuadro se puede apreciar cómo los videojuegos en pocos años han venido a suponer más de la mitad del mercado de entretenimiento audiovisual, superando a la suma de las demás opciones.

Cuadro 2. Reparto del consumo en el entretenimiento audiovisual en 1999, 2005 y 2010.

\begin{tabular}{|l|c|c|c|c|c|c|}
\hline \multirow{2}{*}{ Medio } & \multicolumn{2}{|c|}{1999} & \multicolumn{2}{c|}{2005} & \multicolumn{2}{c|}{2010} \\
\cline { 2 - 7 } & $\begin{array}{c}\text { Millones } \\
\text { de } €\end{array}$ & Porcentaje & $\begin{array}{c}\text { Millones } \\
\text { de } €\end{array}$ & Porcentaje & $\begin{array}{c}\text { Millones } \\
\text { de } €\end{array}$ & Porcentaje \\
\hline Videojuegos & 423 & $22 \%$ & 863 & $35 \%$ & 1.120 & $55 \%$ \\
\hline Cine & 496 & $25 \%$ & 627 & $26 \%$ & 662 & $33 \%$ \\
\hline DVD & 348 & $18 \%$ & 470 & $19 \%$ & 105 & $5 \%$ \\
\hline $\begin{array}{l}\text { Música } \\
\text { grabada }\end{array}$ & 679 & $35 \%$ & 480 & $20 \%$ & 137 & $7 \%$ \\
\hline
\end{tabular}

Fuente: Adese (2003, 2006 y 2011a) y del AMETIC (2011). Elaboración propia. 
Esta preferencia creciente hacia los videojuegos como alternativa de ocio audiovisual se refleja también en que un $53 \%$ de los españoles mayores de 35 años utiliza los videojuegos de manera frecuente o esporádica (Parra et al., 2009). Entre los usuarios de consolas y los PC no existen importantes diferencias en la cantidad de horas que juegan a la semana, como se puede apreciar en el gráfico n. ${ }^{\circ}{ }^{1}$. Los más numerosos son aquellos que dedican entre una y cuatro horas a la semana a esta actividad, a quienes se les denomina medium players (Adese, 2010). Los posibles efectos de este consumo habitual siguen siendo campo abierto y fecundo para la investigación (Levis, 1997; Díaz Soloaga, 2006; Miguel Pascual, 2006).

Gráfico 1: Frecuencia de juego de los gamers de consola y de PC.

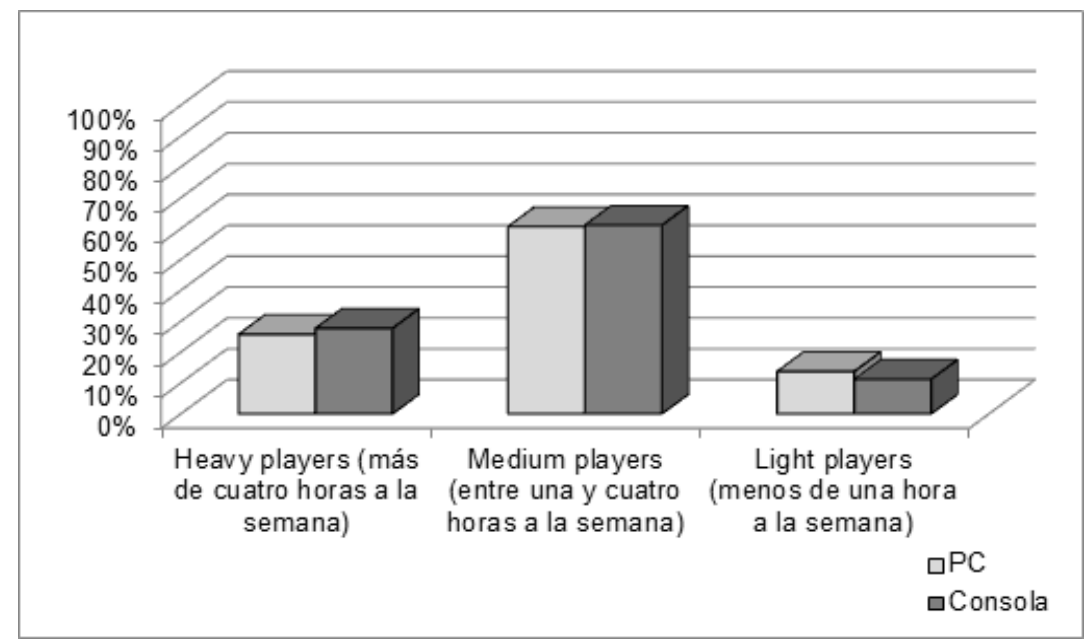

Fuente: Adese, 2010. Gráfico de elaboración propia.

Respecto a las plataformas, los juegos para PC siguen perdiendo cuotas de mercado y en el 2010 representaron apenas un 9\% de la facturación. En cambio la Wii sigue dominando el escenario, con un $44 \%$. Esta consola está teniendo gran aceptación, al conjugar el juego con la competición y el ejercicio físico. Asimismo, el uso de redes está en auge, con juegos en línea que permiten al usuario adecuar el gasto a sus posibilidades, adquiriendo diversas prestaciones a través de micropagos (freemiun).

La plataforma de los móviles sigue perfilándose como la más prometedora. En este sentido, se conjuga la facilidad de que un gran porcentaje de los jugadores habituales posean estos dispositivos, frecuentemente con conexión a Internet, y la ventaja de que los juegos para estos dispositivos suelen requerir de inversiones inferiores para su desarrollo (AETIC, 2011). Son los llamados juegos casuales, que se caracterizan por ser sencillos de producir, fáciles de aprender y que se suelen utilizar en sesiones cortas y repetitivas. 


\section{LA OFERTA NACIONAL DE VIDEOJUEGOS}

El 25 de marzo de 2009 el Congreso de los Diputados aprobó por unanimidad la consideración de los videojuegos como una industria cultural. Esta declaración permitía que las empresas dedicadas a su producción pudieran recibir ayudas y tener un estatus especial en impuestos como el IVA. Los creadores de videojuegos serían considerados a partir de ese momento como artistas (Checa Godoy, 2009).

Sin embargo, España aparece como un mercado con una clara asimetría entre productores y usuarios; el sector de los videojuegos se caracteriza por tener una demanda importante y una producción limitada. Prueba de ello es que la producción española de títulos apenas alcanza un $1 \%$ del total del mercado, frente a la media del 15\% europea. Además, del "top 10" español de ventas del año 2010, ninguno de los juegos ha sido desarrollado por una empresa de este país (Adese, 2010a).

Solo algunos estudios nacionales de pequeña escala producen algo más del $5 \%$ del mercado español y se suelen especializar en el sector de juegos casuales y para móviles (Wyman, 2009). De hecho, los juegos para teléfonos portátiles representan un $56 \%$ del total (AETIC, 2011). La oferta nacional de videojuegos es muy limitada, aunque en términos de demanda este país ocupe un importante lugar a nivel europeo (Wyman, 2009). En un año de gran producción, como fue 2007, solo se realizaron en España unos 150 videojuegos (Checa Godoy, 2009).

El número de empresas desarrolladoras ha pasado de poco más de una docena en el año 2005 a más de 136 en 2010 (Wyman, 2009; DEV, 2010). Pero su facturación es todavía muy baja. Existen excepciones, como Pyro Studios, que ha vendido más de cinco millones de copias de su juego Comandos, convirtiéndose este en uno de los juegos más importantes del mundo. A su vez, FX Interactive alcanzó el millón de copias con su trilogía Imperivm y superó los 100.000 ejemplares con títulos como Runaway 2, Sparta y Sacred (DEV, 2010). Virtual Toys también llegó el millón de ventas en el año 2009 y logró dar salida a cinco millones de copias de tres de sus productos, aunque a través de una empresa francesa (Checa Godoy, 2009). Novarama también ha logrado ocupar un puesto importante en el mercado con su juego Invizimals, distribuido por Sony, que llegó a vender 111.000 copias entre España y Portugal y que sigue estando presente en el ranking nacional de los 50 más vendidos (Adese, 2011a).

Sin embargo, la mayoría de las empresas desarrolladoras de España tiene denominador común el tamaño reducido y la preferencia por la plataforma de los móviles última generación, iPads, iPhones e Internet (DEV, 2010). Dichas plataformas representan una importante alternativa al oligopolio de las compañías distribuidoras, pero una de las principales desventajas de los servicios en movilidad es la excesiva proporción que se queda el operador al realizar los pagos (AETIC, 2011).

Respecto a los videojuegos en línea, algunas páginas web españolas de 
videojuegos han alcanzado importantes cuotas de visitas. La ventaja que presentan es que se ahorran los costes de distribución. Las plataformas de juego congregan distintas alternativas de videojuegos y en parte deben su éxito a su capacidad de establecer redes sociales, apoyándose en la publicidad y en los pequeños pagos por funcionalidades avanzadas (AETIC, 2011).

Otra alternativa por la que optan algunas desarrolladoras españolas de tamaño mediano es realizar trabajos de encargo para las multinacionales, respondiendo a la tendencia que impone este sector hacia la subcontratación y a la externalización de servicios (Wyman, 2009).

Estos modestos logros que se han mencionado se ven condicionados por vida media muy breve de las desarrolladoras españolas. El impulso inicial que tuvieron en los años ochenta y comienzos de los noventa no tuvo una continuidad ni gozó de consolidación cuando se profundizaron las exigencias económicas de las nuevas plataformas y se concentró la producción en Estados Unidos y Japón (Checa Godoy, 2009). El resultado es que los juegos de origen español escasamente suponen un $0,2 \%$ del volumen del negocio global (AETIC, 2011).

En parte, esta situación tiene que ver con la imposibilidad de comercializar este producto al margen de las grandes distribuidoras que monopolizan el mercado, por la falta de apoyos para crear iniciativas nacionales en ese campo. Las grandes distribuidoras exigen a las desarrolladoras los derechos de propiedad intelectual de los títulos producidos y si el videojuego se convierte en un éxito, garantizan ingresos con sus secuelas. De esta forma, se pierden grandes oportunidades de ganancias y se reproduce el estancamiento en una balanza comercial negativa (las importaciones superan las exportaciones) (AETIC, 2011).

Como consecuencia de este panorama, la industria del videojuego en España genera apenas 1.705 empleos, lo que significa apenas un $4,12 \%$ del personal empleado en el sector de contenidos digitales (AETIC, 2011).

\section{LA CONCENTRACIÓN EMPRESARIAL Y SUS ESTRATEGIAS}

En parte, este rezago de la industria nacional es ocasionado por el modelo que rige al sector de los videojuegos, que es el llamado blockbuster o modelo superventas. En él la industria debe afrontar los riesgos de desarrollar un producto costoso cuyo éxito no está garantizado. De modo que las empresas se ven obligadas a diversificar los riesgos para mitigar el efecto de las inversiones realizadas en juegos poco exitosos. Por eso las compañías necesitan tener cierto tamaño, para poder producir la cantidad de juegos que haga posible esa compensación de los fracasos comerciales (Wyman, 2009).

El coste medio de desarrollo de un videojuego para los estudios multinacionales de mayor facturación es de diez a quince millones de euros. El videojuego Halo 2 costó unos treinta millones de euros en 2008 y Grand Theft Auto IV requirió una 
inversión de 80 millones y tardó cuatro años en estar listo (Wyman, 2009). Si se cuentan también las producciones de los estudios de menor facturación, el coste medio por videojuego es de seis millones de euros, cifra todavía muy elevada (Checa Godoy, 2009).

Ante esta necesidad de grandes inversiones, la integración vertical de las empresas del sector se plantea como una importante alternativa para subsistir. Las multinacionales se hacen presentes en muchas o en todas las fases de la cadena de valor para poder alcanzar un tamaño que les permita asumir grandes proyectos con un elevado riesgo. Esa integración vertical actúa, asimismo, en contra de las pequeñas empresas que tienen que recurrir a terceros para la distribución de sus productos (Wyman, 2009).

María Jesús López, cofundadora de Erbe (una de las más importantes desarrolladoras y distribuidoras españolas de videojuegos en la década de 1980), explica que el éxito que tuvieron los juegos elaborados en este país en la época del Spectrum, Amstrad y Comodore remitió porque llegaron las consolas. Con ellas, la necesidad de recursos económicos para desarrollar videojuegos fue mucho mayor y ya no eran suficientes los equipos de trabajo pequeños con los que contaban las empresas punteras de esa etapa. Ahora se necesitan, explica, equipos de alrededor de doscientas personas para poder elaborar un producto que puede tardar en salir dos años al mercado. Además dichos equipos deben ser interdisciplinares, porque tienen que elaborar aplicaciones en línea, efectos especiales, 3D y otros muchos añadidos con los que ni se soñaba hace tres décadas. Según la empresaria, son muy pocas las compañías españolas que pueden asumir ese esfuerzo económico (Retroacción, 2009).

Es por eso que en el mercado español la producción está mayoritariamente en manos de empresas foráneas. Además, los agentes de distribución también se concentran en las multinacionales del sector y a las distribuidoras españolas apenas les queda una reducida cuota (Gfk Emer Ad-hoc research, 2010). Si la distribución es un problema, también lo es la producción, pues, como explica María Jesús López, si el lanzamiento de un videojuego requiere de una inversión tan elevada como una superproducción de Hollywood, tiene que ofertarse necesariamente en el ámbito internacional, porque con el mercado local no podría cubrir esos gastos (Retroacción, 2009). Pero los grandes distribuidores multinacionales colocan el videojuego en la posición de marketing de su particular interés y se llevan una gran parte del precio de venta (AETIC, 2011).

Las tendencias oligopólicas del mercado de los videojuegos también se manifiestan en que los tres principales fabricantes de videoconsolas (Sony, Microsoft y Nintendo) controlan la adjudicación de licencias para la producción de juegos para sus plataformas (Wyman, 2009). Esta concentración empresarial, generada por la necesidad de disminuir el riesgo de fracaso comercial de los grandes lanzamientos, es la causante de que en 2010 apenas cuatro empresas desarrolladoras coparan el 
$44 \%$ de los cincuenta juegos más vendidos en España: Nintendo EAD, Infinity ward, Electronic Arts (EA), Infinity Ward y Konami (Adese, 2011a; cálculos propios).

En las tres primeras multinacionales se ha aplicado la estrategia de integración vertical. Nintendo y Electronic Arts controlan no solo la producción de nuevos juegos, sino la distribución y, en el caso de Nintendo, también la adjudicación de licencias para el software de entretenimiento que utilice su plataforma. Infinity Ward no se queda atrás, pues pertenece a Activision Blizzard, que es un gran distribuidor estadounidense de videojuegos.

Por otra parte, estos grupos empresariales son el resultado de fusiones con otras compañías y compras de empresas más pequeñas, que se efectuaron para mejorar sus ventajas competitivas en el mercado internacional. Activision Blizzard es el resultado de la unión entre Activision y Vivendi Games; Electronic Arts ha comprado desarrolladoras como Criterion y Maxis, para producir grandes éxitos de ventas como Los Sims; Nintendo, por su parte, ha creado y adquirido múltiples empresas que diseñan su software de entretenimiento; y de la misma forma Konami compró a Hudson Soft.

Asimismo, estas multinacionales no se limitan a operar en el sector del videojuego, sino que forman parte de conglomerados que participan en otras actividades mediáticas, como es el caso del Grupo Vivendi, al que pertenece Activision Blizzard; o la franquicia de televisión Chōseishin Series, creada por Konami. También tienen participaciones en otras actividades económicas, como es el caso de Nintendo (equipos de béisbol y baloncesto de Estados Unidos). De esta forma, se puede afirmar que utilizan la estrategia de la integración multimediática y multisectorial.

Adicionalmente, la estrategia de la internacionalización está presente en todos los casos, pues Nintendo (japonesa), EA (estadounidense), Activision Blizzard (también estadounidense) y Konami (japonesa) tienen filiales en América, Europa y Asia, y Nintendo además tiene una en Oceanía. Allí contratan los servicios de empresas de cada lugar y adaptan los contenidos al gusto de los consumidores locales, nacionales y regionales. Es lo que ha hecho EA en Madrid, al instalar su Centro Europeo de Servicios de Desarrollo. La digitalización facilita las economías de escala y la sinergia entre plataformas y productos para aumentar la rentabilidad. Las redes permiten conectar la financiación, la producción y la distribución dentro de cada país y entre países (Castells, 2009).

Es por ello que la oferta se concentra cada vez más en unos pocos actores, que compiten, pero también colaboran, entre sí simultáneamente (Castells, 2009). El mercado del videojuego español es expresión de esta tendencia, como puede verificarse en la lista de los cincuenta juegos más vendidos en este país. Este ranking está principalmente dominado por las multinacionales, aunque existen tres juegos producido por dos desarrolladoras nacionales: en el lugar 30 Invizimals Shadow zone, creado por Novarama y distribuido por Sony (otro grupo multimediático con 
sede nipona y estadounidense); en el 32 Invizimals, del mismo desarrollador; y, en el 47, Planet 51, de Pyro Studios, creadores de otros videojuegos exitosos, como Comandos. No obstante, un $82 \%$ de esos cincuenta juegos más vendidos en 2010 fue producido en Japón y Estados Unidos (Adese, 2011a; cálculos propios).

Tal como explica Moreno (2002), el proceso creativo de los videojuegos en España se dificulta por la fuerte presencia de contenidos extranjeros que se traducen y adaptan al contexto cultural local, ocasionando que la inversión en empresas nacionales se desvíe hacia la distribución de productos masivos ya consolidados. De esta forma, se hace cada vez más necesario el apoyo estatal, por ser la alternativa ante la escasez de inversiones privadas en un sector prometedor y potencialmente capaz de generar empleo.

\section{LOS VIDEOJUEGOS Y LAS AYUDAS PÚBLICAS}

La escasa financiación por parte del Estado es uno de los obstáculos para el crecimiento de la industria española del videojuego. Los nuevos lanzamientos son costosos y presentan muchas incertidumbres sobre la respuesta del mercado. Por esta razón, las desarrolladoras de videojuegos no tienen un fácil acceso a créditos financieros (Checa Godoy, 2009). Sin inversión ni apoyo público, estas empresas no pueden crecer a gran escala, y mucho menos ganar mercado internacional, por lo que se ven relegadas a una posición minoritaria incluso en el consumo nacional.

En lo que se refiere a las ayudas de capital, las que fueron concedidas en 2010 por el Ministerio de Cultura para la modernización, innovación y adaptación tecnológica de las industrias culturales, detalladas en el Boletín Oficial Español de 13 de julio (BOE, 2010), otorgaron al sector de los videojuegos un total de 190.500 euros.

De ese dinero, 67.000 euros fueron adjudicados al desarrollo de videojuegos específicos (por las empresas Abylight, Delirium y Over Top The Games); 30.000 a la implementación de una distribuidora española (Virtual Toys); 73.500 a la actualización tecnológica y comercial de compañías desarrolladoras y 20.000 a la creación de plataformas de difusión y recomendación online de videojuegos (BOE, 2010 y cálculos propios).

Este monto concedido a empresas relacionadas con el videojuego apenas representa un $5,95 \%$ del dinero destinado a estas ayudas. A la industria editorial, por el contrario, se le adjudicó un $18,05 \%$, al cine un $7,91 \%$ y al sector audiovisual un $17,97 \%{ }^{1}$. Sin embargo, estos dos últimos sectores tienen una facturación inferior y menos prometedora que los videojuegos. En el siguiente gráfico se comparan las ayudas recibidas por cada industria.

\footnotetext{
${ }^{1}$ Por la ausencia de clasificación de las ayudas por industria cultural en el BOE citado, se ha definido como industria editorial toda actividad dedicada a la edición y distribución de libros y revistas; como cine la realización de largometrajes, cortometrajes o documentales; y como industria audiovisual la elaboración de productos audiovisuales para televisión, páginas web y publicidad. En los tres casos se han tomado en cuenta las ayudas para actualización tecnológica y para la realización de producciones específicas.
} 


\section{Gráfico 2.}

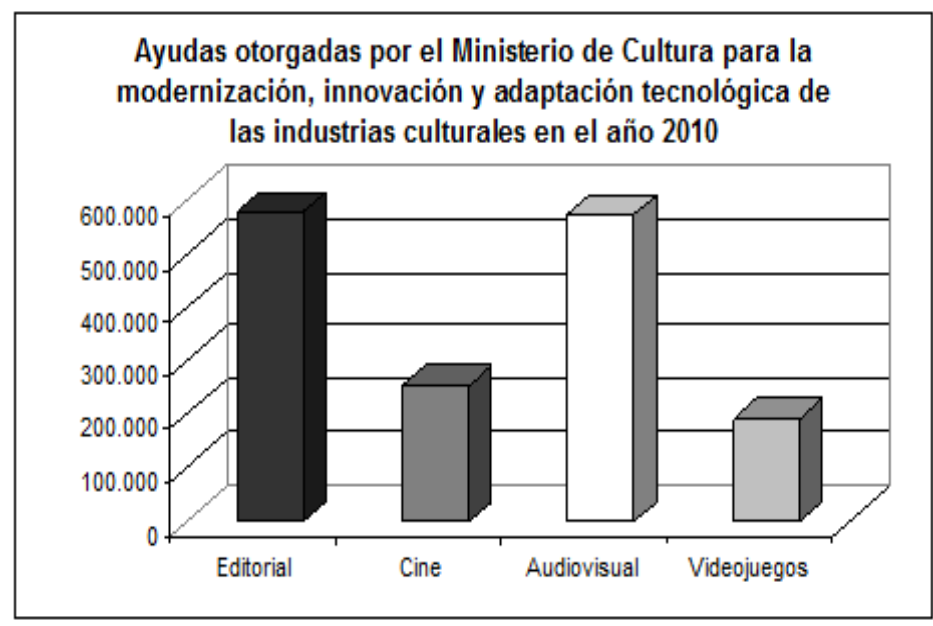

Fuente: BOE (2010). Elaboración y cálculos propios.

No obstante, se han comenzado a manifestar algunas iniciativas de apoyo al videojuego desde el sector público, como son por ejemplo la incorporación explícita de esta industria cultural en el Plan Avanza 2 del Ministerio de Industria, Turismo y Comercio y en el Plan Sectorial del Instituto Español de Comercio Exterior (ICEX). En el primer caso, dentro del eje formativo, que está dotado de un presupuesto de 548 millones de euros, se tienen previstos cursos de programación en realidad aumentada y en realidad virtual aplicada a los videojuegos. Y en el eje de apoyo a la industria de las TIC, ha dispuesto un presupuesto de 130 millones de euros en préstamos y 15 millones en subvenciones para el apoyo de los contenidos digitales (BOE, 2011). Dentro de esta categoría se incluyen los videojuegos, aunque en la convocatoria no se determina el monto mínimo y máximo que será adjudicado a cada tipo de contenido digital.

En lo que respecta al ICEX, ha organizado y financiado en 2011 la participación de empresas de videojuegos made in Spain en la feria Game Connection Europe (París), en la Tokio Game Show (Japón) y en la muestra My Content 2011 (Dubai), con el fin de promocionar la producción nacional (ICEX, 2011).

La ayuda pública a los videojuegos resulta fundamental para un sector en el que se invierte escaso capital-riesgo. Ese apoyo puede ir desde la subvención directa a proyectos de desarrollo de juegos, hasta el equipamiento tecnológico de las empresas, su promoción en el ámbito internacional y la facilitación de la inversión privada mediante créditos concesionales o fondos para servicios previos de estudio (AETIC, 2011).

Un aspecto fundamental dentro de este aspecto es la inversión pública en 
investigación y desarrollo. Los retos tecnológicos que plantea el futuro del videojuego hacen que esta sea indispensable, al igual que la formación de profesionales cualificados.

El estado actual de la inversión en I+D en los videojuegos en España indica que esta se encuentra todavía muy por debajo de lo necesario para que nuestro país pueda convertirse en un referente internacional en producción, con una cuota importante del mercado mundial.

El sector de los contenidos digitales, al que pertenecen los videojuegos, dedicó en 2008 un $1,15 \%$ de su facturación total a labores de investigación y desarrollo. Esta cifra está por debajo del porcentaje que registra el conjunto de la economía española (alrededor de $1,35 \%$ ) y es muy inferior al de las empresas del sector de las tecnologías de la información y la comunicación (TIC), que alcanzan promedios del $5 \%$ de su total de facturación (AETIC, 2010).

En el subsector de los videojuegos se realizó ese mismo año un gasto en I+D de 26 millones de euros, que representa una cuarta parte de la inversión total de la industria de los contenidos digitales. No obstante, esta inversión sufrió una reducción de un $8 \%$ respecto al año anterior (AETIC, 2010), precisamente cuando las exigencias del mercado y los retos tecnológicos, lejos de disminuir, se incrementan significativamente.

En cuanto a la necesaria mejora de la formación profesional, empresas españolas de videojuegos como Enne, Digital leyends y Pyro Studios están colaborando con universidades españolas en la creación de másteres para la formación de desarrolladores de videojuegos. La Universidad Pompeu Fabra de Barcelona fue pionera en esta oferta (http://www.idec.upf.edu/master-en-creacion-de-videojuegos), en el año 2002 (Checa Godoy, 2009). Y recientemente la Universidad Camilo José Cela ha creado el primer Grado en Diseño y Desarrollo de Videojuegos (http://www.ucjc.edu/index.php?section=estudios/titulaciones/titulaciones-oficiales/ titulaciones-grado/ingenieria-multimedia-videojuego). La Universidad de Alicante, por su parte, ha comenzado a fomentar el sector local convocando concursos.

No obstante, el sector adolece de una precariedad laboral que favorece la fuga de cerebros hacia otros países productores de software, capaces de mejorar una oferta de trabajo que se caracteriza por las contrataciones temporales y el pago por obra concluida. Este es otro factor que conduce a la apuesta por contenidos ya consolidados y la escasez de propuestas novedosas (Moreno, 2002).

Finalmente, la piratería es uno de los factores que más afecta la industria del videojuego en España. En este país se duplica el número de descargas ilegales de Estados Unidos y por ello ha sido incorporado a la lista de vigilancia de la Office of the United States Trade Representative y del Caucus Antipiratería Internacional del Congreso de este país. Cerca del $44,6 \%$ de los juegos que se usan en España han sido adquiridos ilícitamente, mediante descargas o a través del top manta. Los videojuegos de pago solo suponen un 1,5\% del mercado online (Adese, 2010). Este elevado índice de piratería crea una mala imagen 
entre productores y distribuidores internacionales, desestimulando asimismo la inversión en el sector.

\section{CONCLUSIONES}

La situación actual de la industria española del videojuego se caracteriza por una configuración oligopólica del mercado, cuya oferta está dominada en gran medida por las multinacionales. Estas se han valido de las estrategias de integración vertical, multimedia, multisectorial e internacional no solo para poder aumentar su rentabilidad, sino para asumir los riesgos significativos que suponen los grandes lanzamientos de videojuegos, que requieren de una inversión superior a los diez millones de euros en muchos casos. Para garantizar el éxito en este contexto, y para internacionalizar la producción, tienen que diversificar los títulos que sacan al mercado y controlar la distribución, lo que complica todavía más las posibilidades de las pequeñas empresas. Estas se ven incapaces de captar una cuota significativa de la demanda nacional y de posicionar sus productos más allá de las fronteras, porque incluso para comercializar sus productos y obtener licencias para el uso de determinadas consolas tienen que recurrir a los "grandes" del sector.

Este panorama no es radicalmente diferente al de otras industrias de ocio audiovisual. Pero las ayudas que esta ha recibido el videojuego para la modernización, innovación y adaptación tecnológica, si se comparan con las del sector editorial, cinematográfico y audiovisual son menores. En I+D ostenta inversiones menores en que el conjunto de la economía española y del sector de las TIC.

No obstante, en la actualidad la demanda española de videojuegos representa más del $10 \%$ de la cuota de facturación de Europa (ONTSI y Neoris España, 2010) y se encuentra en franco crecimiento, siendo uno de los sectores menos afectados por la recesión económica. Si se mejorara la producción nacional hasta hacerla competitiva, este mercado podría significar una importante fuente de ingresos y de generación de empleos. Pero ello pasa necesariamente por la implantación de una clara línea de apoyo gubernamental a esta recién nombrada industria cultural, y dentro de esas ayudas un especial énfasis en la inversión en innovación y desarrollo. Asimismo, convendría aprovechar el repunte del consumo en públicos anteriormente ajenos a este producto (mujeres y personas mayores), ofreciendo planteamientos temáticos distintos y creativos. Además, potenciar el desarrollo de videojuegos casuales y para móviles, que una requieren menor inversión y representan menor riesgo.

\section{REFERENCIAS BIBLIOGRÁFICAS}

AARSET, Espen J.: Cybertext: perspectives on ergodic literature, Johns Hopkins University 
Press, Baltimore, 1997.

ADESE (Asociación Española de Distribuidores y Editores de Software de Entretenimiento): Anuario Adese 2003, 2004, en línea en http://www.adese.es/pdf/anuario-memoria-2003.pdf [enero de 2012].

ADESE (Asociación Española de Distribuidores y Editores de Software de Entretenimiento): Anuario Adese 2005, 2006, en línea en http://www.adese.es/pdf/anuario-memoria-2006.pdf [agosto de 2010]

ADESE (Asociación Española de Distribuidores y Editores de Software de Entretenimiento): Anuario Adese 2009, 2010, en línea en http://www.adese.es/pdf/Anuario2009aDeSe. pdf [octubre de 2010]

ADESE (Asociación Española de Distribuidores y Editores de Software de Entretenimiento): Anuario Adese 2010, 2011a, en línea en http://www.adese.es/pdf/anuario2010/ANUARIO2010.pdf [enero de 2012].

ADESE (Asociación Española de Distribuidores y Editores de Software de Entretenimiento): El videojugador español: perfil, hábitos e inquietudes de nuestros gamers, $2011 \mathrm{~b}$, en línea en http://www.adese.es/pdf/EstilodeVidayvaloresdelosjugadoresdevideojuegos_resumenpresentacion.pdf [enero de 2012].

AETIC (Asociación de Empresas de Electrónica, Tecnologías de la Información y Telecomunicaciones de España): Los contenidos digitales en España 2009, Madrid, 2010, en línea en http://www.aetic.es/CLI_AETIC/ftpportalweb/documentos/Los\%20Contenidos\%20 Digitales\%20en\%20Espa\%C3\%B1a\%202009.pdf [noviembre de 2010].

AETIC (Asociación de Empresas de Electrónica, Tecnologías de la Información y Telecomunicaciones de España): La industria de los contenidos digitales 2010, Observatorio Industrial del Sector de Electrónica, Tecnologías de la Información y Telecomunicaciones, Madrid, 2011.

AMETIC: Informe de la industria de contenidos digitales 2011, Ametic, 2011, en línea en: http://www.cedro.org/docs/documentos/informe_contenidosdigitales2011.pdf?Status=Master [enero de 2012].

AGUADO, Guadalupe; GALÁN, Javier; FERNÁNDEZ-BEAUMONT, José y GARCÍA, Luis José: Organización y gestión de la empresa informativa, Síntesis, Madrid, 2008.

BARAB, Sarah; THOMAS, Michael; DODGE, Tyler; CARTEAUX, Robert; TUZUN, Hakan: "Making learning fun: Quest Atlantis, a game without guns", en Educational Technology Research and Development, vol. 53, n 1, 2005, pp. 86-107.

BARANOWSKI, Tom; BUDAY, Richard; THOMPSON, Debbe; BARANOWSKI, Janice: "Playing for Real: Video Games and Stories for Health-Related Behavior Change", en American Journal of Preventive Medecine, vol. 34, n 1, 2009, pp. 74-82.

BOE (Boletín Oficial del Estado): "Orden CUL/1887/2010, de 23 de junio, por la que se conceden las ayudas a la inversión en capital para promover la modernización, innovación y adaptación tecnológica de las industrias culturales correspondientes al año 2010", en 
BOE, n. ${ }^{\circ}$ 169, Madrid, 13 de julio de 2010, Sec. III, pp. 61783-61082, en línea en http:// www.boe.es/boe/dias/2010/07/13/pdfs/BOE-A-2010-11146.pdf [octubre de 2010].

BOE (Boletín Oficial del Estado): "Resolución de 25 de marzo de 2011, de la Secretaría de Estado de Telecomunicaciones y para la Sociedad de la Información, por la que se efectúa la convocatoria 1/2011 para la concesión de ayudas del Plan Avanza2", en línea en http://www.planavanza.es/LineasEstrategicas/AreasDeActuacion/Impulso/Contenidos/ ContenidosDigitales/Documents/Conv1_2011Avanza2.pdf [febrero de 2012].

CASTELLS, Manuel: Comunicación y poder, Alianza Editorial, Madrid, 2009.

CHECA GODOY, Antonio: "Hacia una industria española del videojuego", en Comunicación, n. ${ }^{\circ}$, vol.1, 2009, pp. 177-188.

CONSALVO, Mia: "Console video games and global corporations. Creating a hybrid culture", en New Media Society, vol. 8, n 1, 2006, pp. 117-137.

DEV (Asociación Española de Desarrolladores de Videojuegos): Directorio de empresas, Madrid, 2010, en línea en: http://www.dev.org.es [enero de 2012].

DÍAZ SOLOAGA, Paloma: "Efectos del uso del videojuegos en niños y adolescentes en España y EEUU", en Icono, 14, n. ${ }^{\circ} 7,2006$, en línea en http://www.icono14.net/revista/ num7/articulos/paloma\%20diaz.pdf [marzo de 2009].

EVANS, Elizabeth: "Character, Audience Agency and Transmedia Drama”, en Media, Culture and Society, vol. 30, $\mathrm{n}^{\circ}$ 2, 2008, pp. 197-213.

GFK EMER AD-HOC RESEARCH: Usos y hábitos de los videojugadores españoles, Adese, Madrid, 2009, en línea en http://www.adese.es/pdf/PPThabitos122009.pdf [agosto de 2010].

GFK EMER AD-HOC RESEARCH: Principales magnitudes de los contenidos digitales en España 2009, Ministerio de Industria, Turismo y Comercio, Red.es y ONTSI, Madrid, 2010, en línea en http://www.ontsi.red.es/contenidos-digitales/articles/id/4280/principales-magnitudes-los-contenidos-digitales-espana-2009-.html [octubre de 2010].

GROS, Begoña; BERNAT, Antonia: "El aprendizaje de competencias de alfabetización digital a través de los videojuegos”, en Aula de Innovación Educativa, vol. 15, n 176, 2008, pp. 12-16.

GRUSIN, Richard: "Dvds, video games and the cinema of interactions", en Ilha do Desterro. A Journal of English Languaje, Literatures in English and Cultural Studies, $n^{\circ} 51,2006$, en línea en http://www.journal.ufsc.br/index.php/desterro/article/viewArticle/9778 [diciembre, 2010].

ICEX (Instituto de Comercio Exterior de España) (2011): Calendario de actividades, Madrid, 2011, en línea en http://www.icex.es/icex/cda/controller/pagelCEX/0,6558,5518394_5519002_5762329_0_0_-1,00.html [febrero de 2012].

INE (Instituto Nacional de Estadística): "Indicadores del sector TIC", en Inebase, 2010, en línea en http://www.ine.es/jaxi/menu.do?type=pcaxis\&path=\%2Ft14/p197/e01\&file=ine- 
base $\& \mathrm{~L}=0$ [octubre de 2010].

HAYES, Elisabeth; GAMES, Ivan: "Making Computer Games and Design Thinking", en Games and Culture, vol. 3, n. ${ }^{\circ} 3-4,2008$, pp. 309-332.

IZUSHI, Hiro; AOYAMA, Yuko: "Industry evolution and cross-sectoral skill transfers: a comparative analysis of the video game industry in Japan, the United States, and the United Kingdom", en Environment and Planning A, vol. 38, $\mathrm{n}^{\circ}$ 10, 2006, pp. 1843-1861.

JOHNS, Jennifer: "Video games production networks: value capture, power relations and embeddedness", en Journal of Economic Geography, vol. 6, n. ${ }^{\circ}$ 2, 2005, pp. 151-180.

KUHN, Kerri-Ann; POPE, Nigel; VOGES, Kevin: "Exploring product placement in video games: an investigation of recall effects", en Proceedings of the Australian and New Zealand Marketing Academy Conference, Dunedin, Nueva Zelanda, diciembre 3-5, 2007, en línea en Queensland University of Technology Digital Repository: http://eprints.qut.edu. au/27714/2/27714.pdf [octubre, 2010].

LEVIS, Diego: Los videojuegos: un fenómeno de masas. Qué impacto produce sobre la infancia y la juventud la industria más próspera del sistema audiovisual, Paidós, Buenos Aires, 1997.

MARTÍ PARREÑO, José: Marketing y videojuegos. Product placement, in-game advertising y advergaming, Esic Editorial, Madrid, 2010.

MIGUEL PASCUAL, Roberto de: "Cuestiones en torno al poderoso efecto de los videojuegos violentos: del neoconductismo a la cognición social", en Icono, 14, n. ${ }^{\circ} 7,2006$, en línea en http://www.icono14.net/revista/num7/articulos/roberto\%20miguel.pdf [marzo de 2009].

MORENO, Pedro Manuel: "El reto del videojuego on line: mercado creciente con escasa industria", en BUSTAMANTE, Enrique (coord.): Comunicación y cultura en la era digital. Industrias, mercados y diversidad en España, Gedisa, Barcelona, 2002.

MINISTERIO DE CULTURA DE ESPAÑA: El cine y el vídeo en datos y cifras, Madrid, 2010, en línea en http://www.mcu.es/cine/MC/CDC/index.html [octubre de 2010].

OKAZAKI, Shintaro; SKAPA, Radoslav; GRANDE, Ildefonso: "Global Youth and Mobile Games: Applying the Extended Technology Acceptance Model in the USA, Japan, Spain, and the Czech Republic", en ZOU, Shaoming (ed.): Cross-Cultural Buyer Behavior, Advances in International Marketing, vol. 18, 2007, pp. 253- 270.

ONTSI (Observatorio Nacional de las Telecomunicaciones y de la Sociedad de la Información) y Neoris España: Informe anual de los contenidos digitales en España 2010, Red.es, 2010, en línea en http://www.red.es/media/registrados/2010-11/1290073066269. pdf?aceptacion=01846117150ff335687adf11501dd406 [noviembre de 2010].

PARRA, David; GARCÍA DE DIEGO, Antonio; PÉREZ, Joaquín: "Hábitos de uso de los videojuegos en España entre los mayores de 35 años", en Revista Latina de Comunicación Social, n. ${ }^{\circ}$ 64, 2009, pp. 694-707, en línea en http://www.revistalatinacs.org/09/ art/855_UCM/56_73_Parra_et_al.html [julio de 2010].

PÉREZ MARTíN, Joaquín; GARCÍA DE DIEGO MARTÍNEZ, Antonio y PARRA VALCARCE, 
David: "Evolución tecnológica e impacto laboral y socio-económico de los videojuegos", en Comunicación, n. ${ }^{\circ}$ 7, vol. 1, 2009, pp. 167-176.

PESTANO RODRÍGUEZ, José Manuel; VON SPRECHER, Roberto; TRENTA, Milena: "Cómics y videojuegos. Dos industrias culturales en conexión”, en Área Abierta, n 25, 2010, en línea en http://revistas.ucm.es/inf/15788393/articulos/ARAB1010130004A.PDF [noviembre, 2010].

RETROACCIÓN (Asociación para el estudio y divulgación de la informática clásica): Mesa redonda "Producción y distribución del videojuego en España: de los pioneros a la industria actual”, Madrid, 2009, en línea en: http://retroaccion.org/mesa-redonda-produccion-y-distribucion-del-videojuego-en-espana-de-los-pioneros-la-industria-actual [febrero de 2012].

SÁNCHEZ, Jaime; MENDOZA, Claudia; SALINAS, Álvaro: "Mobile Serious Games for Collaborative Problems Solving", en Annual Review of Cybertherapy and Telemedicine, vol. 7, 2009, pp. 193-197.

SÁNCHEZ-TABERNERO, Alfonso: Dirección estratégica de empresas de comunicación, Cátedra, Madrid, 2000.

STORZ, Cornelia: "Dynamics in innovations systems: Evidence from Japan's game software industry”, en Research Policy, vol. 37, n. 9, 2008, pp. 1480-1491.

TEIPEN, Christina: "Work and Employment in Creative Industries: The Video Games Industry in Germany, Sweden and Poland", en Economic and Industrial Democracy, vol. 29, n. ${ }^{\circ}$ 3, 2008, pp. 309-335.

UNIVERSIDAD COMPLUTENSE DE MADRID Y ASOCIACIÓN DE VIDEOJUGADORES: Hábitos e iniciación a los videojuegos en mayores de 35 años, 2008, en línea en http:// www.adese.es/pdf/vjadultosestudio.pdf [agosto de 2010].

WYMAN, Oliver: Libro blanco de los contenidos digitales en España 2008, Ministerio de Industria, Turismo y Comercio, red.es y ONTSI, Madrid, 2009, en línea en http://www.red. es/articles/detail.action?sec=228\&id=2660 [octubre de 2010].

ZYDA, Michael: "From Visual Simulations to Virtual Reality to Games", en IEEE Computer, septiembre de 2005, pp. 25-32.

\section{Breve semblanza de los autores}

Vanessa Rodríguez Breijo es Doctora en Ciencias de la Información por la Universidad de La Laguna, con Premio Extraordinario de Doctorado. Ejerce como profesora y vicedecana de alumnos de la Facultad de Comunicación de la Universidad San Jorge. Miembro del Grupo de Investigación en Educomunicación (GIEC), grupo emergente del Gobierno de Aragón. Es autora del libro La televisión y sus audiencias. Un enfoque cultural (2006) y de diferentes artículos científicos. 
José Manuel Pestano Rodríguez es Doctor en Ciencias de la Información y profesor asociado de la Facultad de Ciencias de la Información de la Universidad de La Laguna. Sus líneas de investigación se centran en los usos de las tecnologías emergentes y en los públicos que configuran. También ha desarrollado diferentes investigaciones relacionadas con la radio en la red, el libro digital, los videojuegos y la televisión interactiva, de las que se han publicado artículos científicos y monografías.

(Recibido el 11-07-2011; aceptado el 02-01-2012) 